\title{
Centralidad de actores y bloques en las redes organizativas de movilización en el conflicto por el Plan Regulador Comunal de Peñalolén el año 2011
}

Roberto Cantillan. Universidad de Santiago, Santiago, Chile. José Herrera. Universidad de Chile, Santiago, Chile.

RESUmen | En el Chile de la última década, los análisis de la acción colectiva en episodios de conflicto urbano se han enfocado en los mecanismos cognitivos de subjetivación política que se despliegan en la praxis de los movimientos sociales. De manera complementaria, este artículo se enfoca en los procesos de emergencia de identidades políticas, analizando las dinámicas de influencia y coordinación entre organizaciones voluntarias involucradas en un conflicto particular. Entre los resultados, se destaca la heterogeneidad de los actores más prominentes, pero con una marcada capacidad de intermediación de algunas organizaciones de pobladores. A nivel estructural, el análisis de bloques indica que una dinámica de coordinación de movimiento social se desplegó a través de la interacción de dominios de acción colectiva diferenciados, y que fue esta relación lo que determinó su mayor probabilidad de influencia.

PALABRAS ClAVE | redes, movimientos sociales, conflicto social.

ABSTRACT In the Chile of the last decade, the analysis of collective action in episodes of urban conflict has focused on the cognitive mechanisms of political subjectivation that are deployed in the praxis of social movements. In a complementary way, this article focuses on the emergency processes of political identities, analyzing the dynamics of influence and coordination between voluntary organizations involved in a particular conflict. Among the results, the heterogeneity of the most prominent actors stands out, but with a marked capacity of intermediation of some organizations of settlers. At the structural level, the analysis of blocks indicates that a dynamic of social movement coordination was deployed through the interaction of differentiated collective action domains, and that it was this relationship that determined its greater probability of influence.

KEYWORDS | networks, social movements, social conflict. 


\section{Antecedentes}

Los procesos de neoliberalización desplegados a partir de la década de los ochenta en Latinoamérica han devenido en una alta concentración de la riqueza y el poder (Banco Mundial, 2013 En este marco, el aumento de las desigualdades y de la conflcitividad suele tener como correlato político la activación de procesos de desdemocratización (Tilly, 2000; 2010) y, por lo tanto, de aumento de las constricciones a la influencia popular sobre la política pública. El separatismo social generalizado se instala como tendencia y se hace particularmente evidente en la organización del espacio urbano (Rosanvallon, 2012; Davis, 2014) toda vez que el territorio y sus configuraciones se han convertido en los indicadores más evidentes de las nuevas desigualdades. Al mismo tiempo, los territorios se convierten en los espacios de articulación de identidades políticas emergentes a través de los movimientos sociales (Merklen, 2010; Svampa, 2008).

En Chile, la hegemonía neoliberal articula estructuras relacionales y normativas (Scott, 2011) en configuraciones sociopolíticas que tienden a desarrollar integración social a través del despliegue de mecanismos de mercado (Garretón, 2001). En términos urbanos, desde los años ochenta, la explotación y la licitación competitiva de los recursos presentes en la ciudad (Harvey, 2007) han contribuido a la homogeneización de los espacios urbanos a través de procesos de segregación y gentrificación de las categorías sociales populares (Skewes, 2001).

En términos normativos, la demanda por recursos urbanos queda restringida a la demanda por propiedad privada y, por lo tanto, en la obtención individual de derechos exclusivos y privilegios monopolistas sobre cierta porción de espacio/suelo. La política de vivienda se enfoca así en subvencionar la demanda de consumidores que acceden a este bien a través del mercado (Özler, 2012; Pulgar, 2012). Al mismo tiempo esta ciudadanía propietaria que deja de estar interesada por la política de los bienes comunes, a la vez que se retrae a la formación de identidades en círculos primarios (Castells, 2005; Rosanvallon, 2012). En los últimos treinta años, este ha sido el leitmotiv de los procesos de integración de los pobres urbanos a los recursos producidos en la ciudad (Espinoza, 1988; 1999a; Imilan, 2016).

El régimen chileno ha desarrollado una notable capacidad de integrar el conflicto social: se focaliza en demandas individuales y se reducen las probabilidades de alteración de los programas de asignación de recursos públicos. Para efectos de nuestro argumento, uno de los impactos más relevantes de la lógica del Estado neoliberal ha sido la fragmentación horizontal y vertical de campo asociativo, lo cual ha tenido graves consecuencias para el movimiento popular (Espinoza, 1999a; 2013).

La fragmentación horizontal ha sido descrita como una dinámica de sociabilidad en donde predominan lazos fuertes entre organizaciones homogéneas, en desmedro de la presencia de vínculos débiles entre organizaciones diversas, los cuales pueden mejorar las probabilidades de acceder a recursos escazos e información no redundante. Esta tendencia se ha mantenido y fortalecido en la medida que la política pública enseńa a competir y no a cooperar entre actores, lo cual va en desmedro de las capacidad de cooperación requeridas para la acción colectiva y la emergencia de identidades políticas amplias (Espinoza, 19995, 1999a, 1999b). 
Por su parte, la fragmentación vertical ha sido entendida como la separación entre la política del campo burocrático y la del campo popular. A la radical transformación de la matriz sociopolítica ocurrida durante los noventa, la cual implicó la pérdida de prominencia de actores otrora mediadores con el Estado, como los partidos políticos y los sindicatos (Barozet, 2016), se suma el predominio de mecanismos de participación política no vinculante y de lógicas clientelares (Lomnitz, 2008; De la Maza, 2010; Espinoza, 2013). En términos urbanos: mientras la participación de los actores populares se vio fuertemente restringida, los procesos de redistribución de recursos otorgaron un rol preponderante al sector privado (Gasic, 2018).

El caso chileno, ilustra un proceso en donde el solapamiento de las desigualdes económicas con las desigualdades políticas contribuye funcionalmente a la reproducción de las desigualdades (Oxhorn, 2001). Sin embargo, el modelo neoliberal también contribuye a la emergencia de enclaves de conflicto que abren oportunidades de cambio y de influencia popular sobre la política pública. Un actor constantemente presente en el conflicto urbano chileno ha sido el movimiento de pobladores, el cual, como veremos, emerge como identidad política al activarse modos de coordinación de acción colectiva que amplían la capacidad de movilización de recursos y de definición de fronteras identitarias por parte de las bases populares. Un supuesto básico de esta dinámica es que toda acción potencialmente transformadora emerge de una estructura de relaciones múltiples que sostienen la vida asociativa en el campo popular. De esta manera, la activación de relaciones de cooperación y alianzas en entornos de conflicto político dependen de la certidumbre que ofrece esa estructura de relaciones amistosas o de parentesco (entre otras), construidas a través del tiempo.

\section{Sobre el movimiento de pobladores}

Desde finales del siglo xIx, el movimiento de pobladores ha tenido una presencia histórica casi ininterrumpida dentro del conflicto social chileno, cuestionando parcialmente las estructuras normativas y relacionales que sostienen las desigualdades urbanas (Angelcos, 2016; Espinoza, 1988; Herrera, 2018). Si bien las reivindicaciones de los pobladores se asocian con el campo de reproducción de las condiciones de vida de los trabajadores, se han movido desde la demanda de acceso al suelo y la vivienda, hasta el requerimiento de participación en el cómo se produce la democratización del país. Actualmente, su lucha se ha ampliado al marco del derecho a la ciudad y la vida digna (Angelcos, 2016; Cortés, 2014).

Entre el retorno de la democracia y el año 2010, los pobladores mantuvieron una acción funcionalmente contenida. Notables excepciones a ello han sido las dos tomas de terreno ocurridas en la comuna de Peñalolén los años 1992 y 1998, junto con la fundación del Movimiento de Pobladores en Lucha (MPL) el año 2006 en la misma comuna, el cual se constituyó en una de las organizaciones de pobladores más icónicas de las últimas décadas (Pérez, 2017). La identidad política del movimiento de pobladores reapareció con fuerza en la escena pública en las movilizaciones realizadas entre los años 2010 y 2012 (Pulgar, 2012). Fue en este contexto que surgió la Federación Nacional de Pobladoras y Pobladores (FENAPO), como un 
efecto de agregación de redes de ayuda mutua, motivado por dirigentes locales tras el terremoto de 2010. Estos dirigentes se dedicaron a construir cadenas de articulación de organizaciones de base desde la zona sur y centro del país, siendo la comuna de Peñalolén uno de los nichos más relevantes para esta federación (Pulgar, 2012).

\section{Sobre el caso}

Bajo estas condiciones ambientales, la Municipalidad de Peñalolén presentó un plan de renovación de la estructura normativa que regula el uso del suelo comunal, el Plan Regulador Comunal (PRC), que contemplaba radicales transformaciones en algunos sectores de la comuna. Si bien en un principio había algunos acuerdos sobre los cuales se pudo haber trabajado fructíferamente con la sociedad civil, la Municipalidad no cedió terreno a las demandas de las organizaciones de pobladores y de otros actores afectados, circunscribiendo a su ámbito el control del proceso. El proceso de implementación, al menos en la fase diagnóstica, no fue satisfactorio para el movimiento de pobladores (articulados en torno a comités de vivienda y organizaciones políticas específicas) ni para el resto de la población. Al contrario, varias voces de dirigentes de organizaciones indicaron haber sido "subestimados" y débilmente incorporados en los procesos de discusión. Esto contribuyó de manera importante al conflicto y a la emergencia de identidades políticas antagónicas.

Lo anterior gatilló un proceso de movilización colectiva, que el año 2011 consiguió el desarrollo de un plesbicito comunal popular respecto a la aprobación o rechazo del Plan Regulador. Hasta ese momento, había antecedentes de procesos de consulta comunal que habían sido desplegados desde abajo, los cuales se desarrollaron en sectores de alta capacidad adquisitiva. Ejemplo de ello fue el caso de Vitacura en el sector oriente de Santiago (Poduje, 2008).

A modo de síntesis, cabe destacar tres elementos de la contienda política que convergen en este episodio de conflicto: El contexto, marcado por las algidas manifestaciones estudiantiles y procesos organizativos emergentes en comunas populares como Peñalolén. Actores enfrentados en torno a la necesidad funcional de modificar el PRC planteada por la Municipalidad de Peńalolen y, un modo de coordinación de acción colectiva activado por organizaciones populares para enfrentarse a sus oponentes del gobierno comunal. La importancia de esta movilización descansa no solo en el amplio nivel de convocatoria y masividad alcanzado, sino también en lo inesperado de su efectividad. ${ }^{1}$ No obstante, persiste un desconocimiento respecto a cómo se configuraron las redes de asociatividad sobre las cuales emergió este movimiento. Indagar estos claroscuros permite conocer más sobre un proceso a través del cual la sociedad civil chilena se hizo, en tiempos recientes, parte activa de la disputa por el poder político y social.

Por ende, el presente análisis fue guiado por la siguiente pregunta: ¿Cómo se configuró la red de movilización de organizaciones de la sociedad civil de Peñalolén y, en específico, qué actores fueron los más centrales en el conflicto y cómo afectaron sus posiciones al entramado mayor? 


\section{Marco teórico}

Este artículo se realizó a partir del supuesto, según el cual, el cambio social se explica mejor a partir del entendimiento de las propiedades emergentes de las estructuras relacionales sobre las cuales los sujetos dan sentido a su acción. Esta clave de análisis nos sitúa en un plano realista y fructífero, por dos grandes razones: i) Considera que las interacciones que activan los actores colectivos en entornos de conflicto para elaborar nuevas estructuras en contradicción con las actuales, son, a su vez, dependientes estructuralmente de relaciones múltiples que se configuran a través del tiempo (Embebdeness o incrustación en contextos multiplexados), en distintos dominios de la vida social (Granovetter, 1985; White, H., 2008).

Y ii), que esta perspectiva nos permite superar la dicotomía analítica entre lo social y lo político, que tiende a comprender reificadamente los procesos de la política popular y la liberal demócrata (Tilly et al., 2005).

\section{Desigualdad urbana}

La ciudad es un campo de estructuras normativas y relacionales que emerge como tal a partir del conflicto por recursos entre actores colectivos y la hegemonía resultante de modos culturales. De este modo, analizar la política de la desigualdad en la ciudad implica poner atención en los patrones de estabilidad y de cambio, a través del análisis de las redes que crean y distribuyen la riqueza en ella (White, D. R., 1994). De este modo, las luchas por la ciudad serán entendidas como episodios de conflicto (Tilly et al., 2005) en los cuales surgen interacciones que perturban los patrones de interacción entre Estado y ciudadanía, posibilitando la creación y el cambio social. Todo en función de demandas de actores particulares interesados por influir sobre la política de regulación de los recursos urbanos.

Aun cuando las clases dirigentes se movilizan con más eficacia y disfrutan de un acceso más directo a los instrumentos del poder estatal, y que la actuación de los Estados se encamina habitualmente a fortalecer las desigualdades categoriales existentes (Tilly, 2000), es en estos episodios donde las categorías subordinadas de la población construyen redes que les permiten articularse en torno a identidades políticas conflictivas. Es en este sentido que el poder (Burt, 1977; Scott, 2007) puede ser entendido como la expresión de la capacidad posicional de las clases populares de aprovechar oportunidades para instalar y cumplir sus objetivos políticos, pese a las restricciones impuestas.

\section{Sociedad civil y movimiento social}

En el amplio campo de la política pública (Tilly, 2010) convergen dos subcampos: La sociedad civil, que contiene a todas las organizaciones voluntarias involucradas en la promoción de la acción colectiva y la producción de bienes colectivos (Crossley, 2010; Diani, 2015). Y, el campo de organización burocrático. Para estudiar estos campos de acción, se debe poner especial atención en la configuración de los vínculos que conectan a los miembros de los campos, y en los patrones de interacción entre campos y subcampos (Diani, 2015). La acción colectiva enmarcada en estos contextos es entendida como una instancia política de creación y cambio social, que involucra 
a distintos tipos de actores, incluyendo siempre a los gobiernos (Tilly, 2010). Los movimientos sociales, en efecto, son mejor comprendidos como "modos de coordinación" de acción colectiva (Diani, 2015) particulares, que se despliegan dentro de procesos sociales más amplios. Por 'modos de coordinación' nos referimos aquí a los procesos relacionales a través de los cuales se asignan recursos y definen límites identitarios dentro de una cierta colectividad de organizaciones formales e informales

Desde esta perspectiva, el movimiento social es: i) un modo de coordinación de acción colectiva constituido por densas redes informales de organizaciones voluntarias que comparten ii) una identidad colectiva que trasciende a una campaña o evento específico, adoptando objetivos de causas más amplias (Diani \& Bison, 2004). Lo anterior implica que este modo se configure en torno a un intercambio intenso de recursos y un trabajo, igualmente intenso, de definición de límites identitarios, lo cual implica el reconocimiento claro de un oponente político. Otros modos de coordinación son: El modo coalicional, que presenta bajos niveles de cohesión y está circunscrito a coyunturas; por lo tanto, no existe como actor que trasciende al evento en cuestión. Un modo comunitario, representa una dinámica con una identidad que trasciende al evento, pero que está arraigada en comunidades y territorios particulares. Y un modo organizacional, el cual se despliega en torno a un muy bajo nivel de intercambio de recursos y un muy leve trabajo de fronteras identitarias. (Más detalles en Diani, 2015).

El análisis realizado aquí se centra en la estructura de las redes interorganizacionales a partir de una evaluación de su densidad y conectividad, considerando datos contextuales.

\section{Centralidad y poder}

Coherente con la perspectiva de análisis adoptada, nuestro objetivo será la búsqueda de patrones de interacción entre actores y modos de coordinación de acción colectiva; y, en específico, detectar la configuración relacional de dinámicas de movimiento social y su importancia para el devenir de los conflictos (Diani, 2003, 2004, 2015). Para esto, es importante indagar en las propiedades de localización que hacen a algunos actores o grupos más prominentes que otros y, en efecto, más influyentes. De esta manera pretendemos aportar en la descripción de patrones de configuración que facilitan la emergencia de identidades políticas, las cuales, para desarrollar influencia, entran en dinámicas de disputa y colaboración con otras identidades (Tilly, 2000; White, H. C., 2008). El supuesto fundamental es que las actitudes de las personas y grupos se forman típicamente en entornos interpersonales (e interorganizacionales) en los cuales las posiciones influyentes en ciertos temas están en desacuerdo y son susceptibles de cambio. Por lo tanto, se usarán medidas de centralidad que nos permitan analizar estas propiedades de manera relacional y pragmática (Friedkin, 2006; Knoke, 1994; Knoke \& Burt, 1983; Wasserman \& Faust, 2013).

El segundo supuesto es que la probabilidad de influencia política aumenta en proporción directa al grado de intermediación, flujo y prestigio (indegree). En efecto, las identidades son más poderosas en la medida en que se vuelven influyentes a través del desarrollo de capacidades para transformar recursos en influencia (Burt, 
1977). En escenarios de conflictos políticos, los actores influyentes son los intermediarios que gozan de buena reputación y prestigio. Por consiguiente, es fundamental detectar actores que conectan e integran dominios lejanos, ya sea por diferencias ideológicas, por afiliaciones incompatibles, o por distancias territoriales (Gould \& Fernandez, 1989). Todas las medidas de centralidad aquí usadas evalúan, por lo tanto, la distribución probable de la influencia dentro de la red de movilización (Wasserman \& Faust, 2013).

\section{Metodología}

La metodología que adoptamos supone que una estructura relacional es un espacio multidimensional de posiciones o lugares sociales, entre los cuales los miembros de una población están distribuidos de manera desigual no aleatoria (Scott, 2011). El análisis de las redes sociales se centra en el estudio de estas estructuras reticulares emergentes (Carrington, Scott, \& Wasserman, 2005), tomando como unidades de análisis los nodos y los vínculos que se configuran en entornos de competencia y/o colaboración.

El análisis estadístico que se usará es de tipo sociométrico y no paramétrico. Los análisis sociométricos, a diferencia de los métodos paramétricos, son más robustos y no se basan en el supuesto de que los datos se extraen de una distribución normal de probabilidad dada. Así, los datos devienen de: i) observaciones dependientes, y ii) con distribuciones anormales. Por consiguiente, es menester indicar que generalmente se trabaja con iii) poblaciones y no muestras (Borgatti, Everett, \& Johnson, 2018).

Para el análisis de actores consideramos relaciones de cooperación direccionadas ${ }^{2}$ y no direccionadas; los nodos son las organizaciones de la sociedad civil de Peñalolén detectadas. Adicionalmente, para el análisis de bloques, consideramos relaciones (direccionadas y no direccionadas) de: i) cooperación, ii) confianza, iii) flujo de recursos, iv) valores compartidos. Este procedimiento hace posible la emergencia de estructuras más realistas de coordinación, a la vez que permite interpretaciones más sustantivas respecto de los procesos organizativos (Doreian, Batagelj, \& Ferligoj, 2005; Wasserman \& Faust, 2013).

\section{Datos}

La investigación que circunscribe este artículo es de tipo mixto (Domínguez \& Hollstein, 2014). Por lo tanto, durante el trabajo de campo se aplicaron cuestionarios $(\mathrm{N}=31)$ y entrevistas cualitativas $(\mathrm{N}=12)$, a lo largo de los ańos 2017 y 2018, instrumentos que nos permitieron identificar setenta organizaciones involucradas en el conflicto. Si bien el foco del presente está en un análisis sociométrico, se utilizaron datos contextuales derivados de la información producida a través de la aplicación de entrevistas en profundidad, para complementar algunas ideas derivadas de los análisis sociométricos.

2 Con la excepción de la medida de grado de entrada (indegree), todas las demás medidas se realizan considerando matrices simetrizadas. 
A través del cuestionario se aplicó un generador de nombres en donde los entrevistados podían nombrar hasta cinco organizaciones con las cuales cooperaron de manera cercana durante los ańos en los cuales se desarrolló el conflicto (2009-2011). Este límite de cien menciones está fundamentado en el objetivo de distinguir a los actores más prominentes en términos de prestigio (indegree) en contextos multirrelacionales. Esto ya que esta técnica permite, además, generar datos sobre otras relaciones en las cuales están incrustadas las relaciones de cooperación.

La estrategia de muestreo utilizada estuvo constituida por dos fases complementarias: i) el primer paso fue identificar los tipos de organizaciones involucradas en el proceso contencioso, ya sea que estuvieran a favor, indiferentes, o en contra de la modificación del PRC; ii) para luego iniciar un proceso de bola de nieve, con una orientación nominalista / etic (Borgatti, Everett, \& Johnson, 2018), lo cual implica que los límites fueron definidos por los investigadores siguiendo un criterio de saturación. En efecto, se entrevistó a actores calificados con un empate con cualquier actor ya seleccionado, en olas sucesivas hasta que se repitió el $40 \%$ de los nombres ya mencionados. Sumado a esto, una vez terminado el proceso de aplicación de cuestionarios, se complementó con la aplicación del mismo instrumento a organizaciones que no aparecen mencionadas pero que, dada la revisión de fuentes secundarias (periódicos municipales), resultaron ser relevantes.

\section{Objetivo y operacionalización}

Nuestro objetivo fue "analizar la centralidad de los actores y bloques para comprender las dinámicas de influencia e identidad en la estructura de cooperación en el proceso de movilización de las organizaciones sociales".

De esto, se desprenden dos subdimensiones, con sus correspondientes indicadores, definiciones y medidas: centralidad de actores y centralidad de grupos.

\section{Centralidad de actores}

a. Centralidad de grado de entrada (indegree): El grado de entrada de cada actor se denota por: $d_{i}\left(n_{i}\right)$. Esta medida expresa el número de nodos adyacentes a $n_{i}$ (Wasserman \& Faust, 2013). La idea es que los actores que son prestigiosos tienden a recibir muchas nominaciones o elecciones. La medida estandarizada es:

$$
P_{D}^{\prime}\left(n_{i}\right)=\frac{X_{+i}}{g-1}
$$

b. Centralidad de intermediación: Esta medida describe la probabilidad de que una comunicación entre un actor j y un actor $\mathrm{k}\left(g_{j k}\right)$ tome una ruta particular, entre todas sus geodésicas (utilizando los caminos más cortos para llegar a otro actor) $1 / g_{j k}$. En particular, nos interesa detectar la probabilidad de que un actor distinto, i, esté implicado en la comunicación entre los dos actores. Sea entonces $g_{j k}\left(n_{i}\right)$, el número de geodésicas que unen a los dos actores y que contienen al actor i. Esta probabilidad se estima como $g_{j k}\left(n_{i}\right) / g_{j k}$ suponiendo que todas las geodésicas tienen la misma probabilidad de ser elegidas para la comunicación, y que se va a optar por el camino más corto. El índice, por lo tanto, es una suma 
de probabilidades (Wasserman \& Faust, 2013). Tiene un valor mínimo cuando $n_{i}$ no se coloca en ninguna de las geodésicas. Y su máximo se alcanza cuando el actor i-ésimo se coloca en todas las geodésicas. Su expresión estandarizada es $\left(\mathrm{C}_{\mathrm{B}}^{\prime}\right.$ Centrality betweenness):

$$
C_{B}^{\prime}\left(n_{i}\right)=C_{B}\left(n_{i}\right) /[(g-1)(g-2) / 2]
$$

c. Centralidad de flujo (Bolland, 1988): Dado que la anterior medida no considera que puede haber actores "expansivos" y, por lo tanto, que algunas geodésica tendrán más probabilidades de utilizarse como caminos, la centralidad de la intermediación del flujo normalizado considera probabilidades desiguales y proporciona una medida que considera todos los caminos posibles entre dos actores, y no solo las geodésicas.

$$
C_{C F(i)}^{\prime}=\frac{v_{(i)}}{\max \left(v_{*}\right)}
$$

d. Centralidad beta (Bonacich, 1987): Este enfoque considera que la centralidad es una función de cuántas conexiones tiene uno y los actores en relación con uno. Así, el poder y la centralidad de un actor depende de la centralidad y poder de otro de manera simultánea. En efecto, el parámetro $\beta$ (que es elegido por el usuario) sirve como un peso basado en la longitud. Por lo tanto, la suma de la serie proporciona el número total de caminatas entre cada par de nodos de todas las longitudes posibles, ponderados por $\beta \mathrm{k}-1$. Si damos un peso positivo $(0,5)$ al parámetro, analizaremos entonces la centralidad, mientras que si asignamos un parámetro negativo $(-0,5)$, analizaremos las relaciones de poder (o de dependencia). En este caso, usamos un parámetro positivo.

$$
C(\alpha, \beta)=\alpha(I-\beta R)^{-1} R 1
$$

\section{Detección y centralidad de bloques}

a. Densidad grupal: Grado de conectividad hacia dentro del grupos.

$$
D=\frac{\text { Conexiones efectivas }}{\text { Conexiones potenciales }}
$$

b. Centralidad de grado grupal (Everett \& Borgatti, 1999): Definimos la centralidad del grado del grupo como el número de nodos no grupales que están conectados a los miembros del grupo. Esto se puede normalizar considerando el tamaño del conjunto de miembros que no pertenecen al grupo. Si C es un grupo que es un subconjunto del conjunto de vértices $\mathrm{V}$, entonces denotamos con $\mathrm{N}$ (C) el conjunto de todos los vértices que no están en $\mathrm{C}$, pero que son adyacentes a un miembro de C.

$$
C G G=\frac{|N(C)|}{|V|-|C|}
$$


c. Detección de bloques: Ecuación para cálculo de coeficiente de correlación producto-momento de Pearson, para relaciones múltiples direccionadas, como medida de equivalencia estructural. Usamos esta medida para detectar bloques de organizaciones equivalentes estructuralmente.

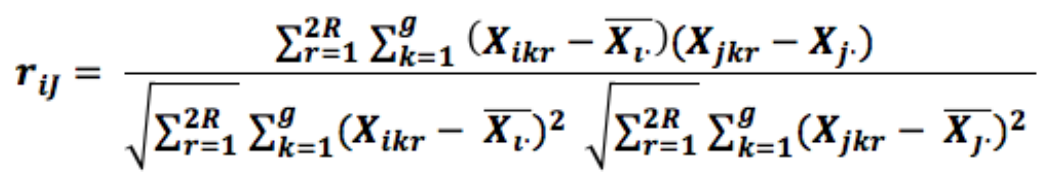

Hipótesis

1. Las medidas de centralidad de actores se distribuyen de manera desigual, y se concentran en los actores del movimiento de pobladores.

2. Los actores más centrales son parte de bloques altamente cohesionados (con mayor densidad).

3. El bloque más central y denso agrupa a las organizaciones de pobladores, siendo posible describirlo como un modo de coordinación de movimiento social

\section{Procedimientos}

Todos estos análisis fueron realizados con el programa Ucinet v. 6 y NetDraw (Borgatti, 2002; Borgatti, Everett, \& Freeman, 2002;).

1. Primero, se estiman y comparan los puntajes de centralidad por cada actor, y se realiza un análisis de correlación de Spearman entre los puntajes de intermediación, flujo y prestigio (indegree) de cada actor.

2. Se estiman bloques equivalentes en términos estructurales a través del procedimiento $\mathrm{CONCOR}^{3}$ (análisis de convergencias de correlaciones iteradas) (Wasserman \& Faust, 2013), usado para detectar actores estructuralmente equivalentes en función de las pautas relacionales. El procedimiento hace uso del estadístico Producto-momento de Pearson para detectar correlaciones significativas entre matrices y conformar bloques. Las relaciones consideradas son: a) flujo de recursos, b) valores compartidos, c) confianza, y d) Cooperación.

3. Se estiman los puntajes de centralidad y la densidad para cada uno de los bloques.

\section{Resultados y análisis}

Con la modificación al PRC, el movimiento de pobladores evaluaba una merma en sus posibilidades de acceder a suelo para la construcción de viviendas sociales en la comuna de Peńalolén. Por su parte, las comunidades de base visibilizaban peligros relacionados con la densificación de los espacios, la segregación y posibles expropiaciones. A grandes rasgos, estos fueron los factores que condicionaron el despliegue de un marco de incertidumbres que facilitaron el despliegue de campañas antagónicas.

3 Puede ser considerado como un método divisor de clústeres jerárquicos. 
Las modificaciones consideraban el cambio de uso de suelo en algunas zonas altamente valoradas por los vecinos de barrios populares. Al respecto, tres propuestas de modificaciones parecieron especialmente conflictivas: i) la construcción de edificios de doce pisos en la franja Américo Vespucio; ii) la expropiación de terrenos ubicados en la quebrada de Vitor; y iii) la falta de terrenos para la construcción de viviendas sociales. A estos temas se agregaron otros igual de controversiales. Por ejemplo, grupos de distinto carácter y generalmente antagónicos a los de pobladores, como fue el caso de la Comunidad Ecológica, también vieron afectados sus intereses de mantener bajo su control y cuidado el corredor biológico en que está emplazada la comunidad. En efecto, a lo largo del proceso de movilización, la Comunidad Ecológica, representada por su Junta de Vecinos (JJ.Vv.), se posicionó finalmente en contra del PRC municipal, afectando de manera importante el devenir del conflicto.

Es así como se lograron articular en torno a una demanda diversos dominios de red emplazados en distintos sectores de la comuna: i) las JJ.vv.; ii) grupos culturales en sectores emblemáticos; iii) grupos de base dedicados a la educación popular; y iv) el mundo de acción colectiva estrictamente ligado a la demanda por el territorio y la vivienda. Como veremos, si bien fue el modo de coordinación de movimiento social uno de los bloques más importantes en las acciones desplegadas, fueron la coordinación y la efectividad en la activación de redes populares de distintos tipos de organizaciones cívicas, arraigadas a un extenso y heterogéneo territorio, los factores que posibilitaron la formación de alianzas estratégicas con límites categoriales amplios pero muy definidos, facilitando la polarización y el despliegue de campańas antagónicas.

Un aspecto clave necesario de considerar es la presencia protagónica de una organización paraguas de la FEnAPO, el Movimiento de Pobladores en Lucha (MPL). Esta organización había logrado posicionar dentro de la Municipalidad a un personaje de suma relevancia, Lautaro Guanca, dirigente de la organización y concejal municipal, lo cual permitió transacciones importantes para el movimiento: hubo acceso a información y, por consiguiente, mayores capacidades para la fiscalización por parte de las organizaciones de base -que tenían contacto con el MPL y el Partido Igualdad-. ${ }^{4}$ Esta "concejalía popular" (como era llamada por el movimiento) fue un actor influyente debido al papel fiscalizador y el esfuerzo que desarrolló para traducir la complejidad técnica de las modificaciones propuestas por la Municipalidad.

\section{Sobre la centralidad de los actores}

Para este análisis, elegimos considerar las medidas de centralidad beta en su espectro positivo, es decir, excluyendo la medida de poder. Por su parte, nos pareció importante medir el prestigio de los actores a través del grado de entrada, en tanto esta cualidad emergente es relevante, toda vez que los actores centrales que buscan ser influyentes pueden serlo en la medida de que gocen de credibilidad y reconocimiento (Knoke, 1994). También, siguiendo la recomendación de Bolland (1988), elegimos incluir la medida de centralidad de intermediación junto a la de flujo. 
Cabe precisar que gran parte del análisis se centró en las quince organizaciones más prominentes, según se muestra en los resultados de la tabla 1 .

Según se recoge en esa primera tabla, los actores más relevantes son: i) comités de vivienda (CV); ii) organizaciones de educación popular (ОРв); y iii) organizaciones políticas de pobladores (OPP). Estas últimas, cabe indicar, son expresiones de diásporas afines de comités de vivienda y entidades asesoras. El MPL ( $\left.n^{\circ} 38\right)$ es la organización con el mayor grado de centralidad; considerando todas las medidas, es una organización que, además de estar fuertemente enlazada con su entorno cercano, tiene una gran influencia en las organizaciones que desarrollan otras dinámicas de cooperación y que tienden a ser más periféricas en la red de movilización; entre ellas, por ejemplo, aquellas que se dedican a la educación popular en entornos locales, las organizaciones de tipo cultural y las de gobierno de base. Esto posibilitó el asesoramiento técnico y, en general, el acceso rápido a recursos valiosos para contiendas de este tipo por parte de instancias organizativas donde generalmente escasean los recursos. Esto es verificable si consideramos su puntaje de prestigio (Indeg=18,8), de intermediación $(B=10)$, y de Centralidad Beta (Beta=0,4).

Una cuestión importante es comprobar si efectivamente el prestigio, según indica la teoría, varía en proporción directa a la centralidad de intermediación. $\mathrm{O}$, dicho de otra manera, resulta importante de comprobar si acaso el rol de una organización prestigiosa tiene una relación directamente proporcional con una posición de intermediación. Al respecto, y considerando supuestos enunciados en el marco teórico, se realizó un análisis de correlación de Spearman, mediante el cual se puede inferir una relación proporcionalmente directa con una fuerte magnitud entre el puntaje indegree y el puntaje de flujo (Rho de Spearman de 0,776, p. <0,000), y el indegree con el puntaje de intermediación (Rho de Spearman de 0,756, p. <0,000). Así, en este caso, los actores que son mejores intermediarios tienden a ser los más prestigiosos.

\begin{tabular}{|c|c|c|c|c|c|}
\hline ID & TIPO & NINDEG & NBETWEENNESS & NFLOWBET & NBETACENT \\
\hline 38 & OPP & 18,841 & 10,09798813 & 10,2291136 & 0,492 \\
\hline 34 & OPB & 10,145 & 2,875969172 & 2,77848816 & 0,011 \\
\hline 5 & CV & 8,696 & 3,651351929 & 0,91716397 & 0,470 \\
\hline 25 & OC & 8,696 & 1,76668489 & 0,77756464 & 0,010 \\
\hline 36 & OPB & 7,246 & 4,66675806 & 4,17945433 & 0,007 \\
\hline 21 & OC & 7,246 & 1,488602638 & 1,27450979 & 0,010 \\
\hline 57 & OV & 5,797 & 2,09830308 & 1,87411201 & 0,003 \\
\hline 58 & OV & 5,797 & 1,023525357 & 0,72144073 & 0,003 \\
\hline 49 & OV & 4,348 & 7,555413246 & 7,59271097 & 0,078 \\
\hline 9 & CV & 4,348 & 7,114186287 & 7,32665539 & 0,269 \\
\hline 13 & CV & 4,348 & 0,103012219 & 0,63974142 & 0,247 \\
\hline 28 & Otros & 4,348 & 1,257459521 & 1,03367436 & 0,029 \\
\hline 20 & OC & 2,899 & 1,278772354 & 1,30008531 & 0,005 \\
\hline 14 & Otros & 1,449 & 3,367434025 & 3,11878371 & 0,019 \\
\hline & Centralización & $16,257 \%$ & 9,30 & 9,618 & - \\
\hline
\end{tabular}

TABLA I | Puntajes de centralidad. Aparecen representados los quince actores más prominentes junto a indicadores de centralización

FUENTE: ELABORACIÓN PROPIA 
La intermediación del MPL ( $\left.\mathrm{n}^{\circ} 38\right)$ posibilita que otras organizaciones con un fuerte arraigo en territorios y comunidades específicas $-\mathrm{y}$ que, por lo tanto, suelen ser periféricas en entornos mayores en relación a las que tienen mayor diversidad de contactos- puedan acceder de manera indirecta a recursos valiosos disponibles en otros entornos, como los propios del campo burocrático. También, se hace posible el acceso a información especializada, a través de los lazos que tienen organizaciones como el MPL o FENAPO con entidades universitarias y profesionales.

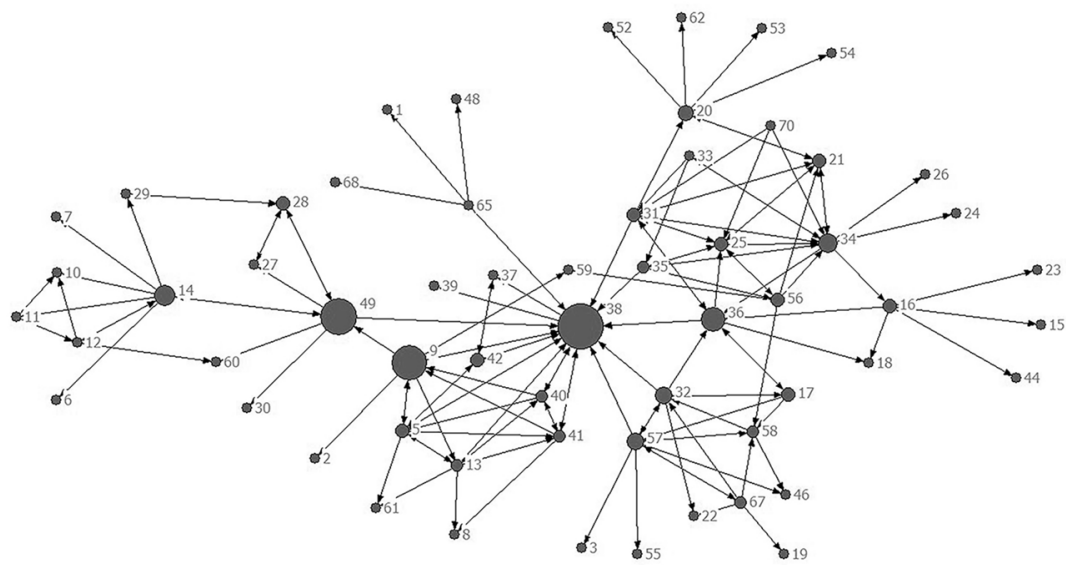

Figura I | Red de cooperación organizaciones de Peñalolén. Los nodos se diferencian por tamaña en función de su grado de intermediación (Betweenness)

FUENTE: ELABORACIÓN PROPIA

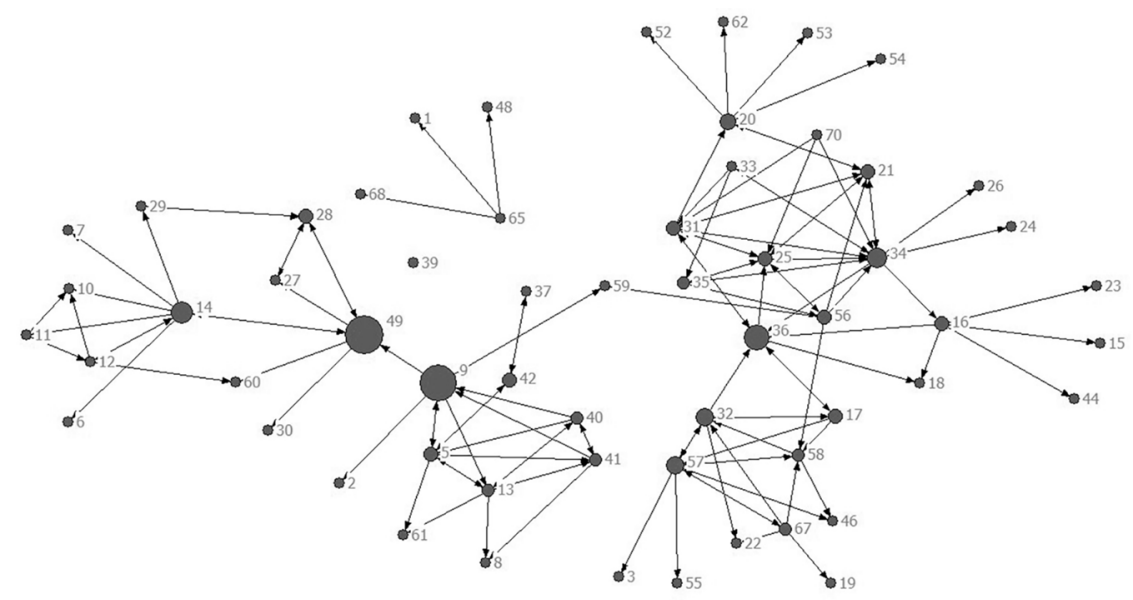

FIGURA 2 Red de cooperación sin el actor más prominente. Los nodos se diferencian en tamaño por su grado de intermediación

FUENTE: ELABORACIÓN PROPIA 
Otra tendencia importante que reflejan las medidas de prestigio es que las organizaciones de tipo cultural (oc) ( $\left.\mathrm{n}^{\circ} 25\right)$, y las dedicadas a la educación popular (орB) $\left(n^{\circ} 34\right)$ son también relevantes, aunque influyentes en su entorno local en desmedro de la red total, puesto que los puntajes de intermediación y de centralidad Beta no parecen reflejar una relación con el prestigio. Sin embargo, es importante destacar que estas organizaciones son más relevantes que las de gobierno de base (ej. JJ.vv.), ya que actúan como enlace entre las organizaciones del movimiento de pobladores (las más centrales) y los territorios específicos en poblaciones emblemáticas de la comuna.

Si bien no cuentan con altos puntajes de intermediación, las organizaciones dedicadas a la educación popular (орв) y las de tipo cultural (OC) son altamente prestigiosas, lo que difiere de las tendencias revisadas. Esto permite indicar que son parte de conglomerados densos, y con pocas conexiones estratégicas que les permitan acceder a recursos diversos. Ello también es indicador de los límites de la trama organizativa de las poblaciones emblemáticas, siendo en estos circuitos donde las identidades políticas de las poblaciones se sostienen y hacen persistentes a través de prácticas comunitarias (como las opв), a la vez que son el soporte de la continuidad de la cultura de los pobladores. Esto es posible de corroborar si consideramos que son organizaciones de este tipo las que sostienen las celebraciones de los aniversarios de los diversos sectores de Lo Hermida y La Faena. En breve, en términos de integración horizontal, estas organizaciones son un bloque que asegura el flujo de información entre el centro y la periferia de la red, además de movilizar entornos locales no asociados con organizaciones de vivienda y/o sus organizaciones políticas. Profundizaremos en esto en la segunda parte del análisis.

Los actores más influyentes en términos de intermediación pertenecen a distintos tipos, haciéndose muy relevantes organizaciones de tipo vecinal, como la JJ.Vv. de la Comunidad Ecológica ( $\left.\mathrm{n}^{\circ} 49\right)$. Si miramos la red completa (figura 1 y figura 2 ), podemos corroborar que este actor, además de ser un importante punto de corte, posee un variado espectro de accesos a distintos tipos de actores y recursos. Sin ir más lejos, su empate con organizaciones gremiales y especializadas en asesoramiento técnico $\left(\mathrm{n}^{\circ} 27,28,30\right)$, además de su histórico y positivo vínculo con la Municipalidad, le dan la legitimidad suficiente para actuar como un nodo altamente influyente. En este mismo sentido, sus vínculos directos con el Consejo de Movimientos Sociales ${ }^{5}$ y su importancia estratégica como aliado para la Municipalidad y el movimiento de pobladores, le permiten estar intermediando entre los bloques antagónicos, controlando flujos de información técnicamente relevante, a la vez que su compromiso con la voluntad de frenar el nuevo PRC le permite ser un actor que motiva el encuentro y el diseño de propuestas innovadoras. Su rol facilita, en este caso, la influencia popular sobre la política pública.

Por su parte, la Municipalidad ( $\left.\mathrm{n}^{\circ} 14\right)$ aglutina poder en tanto tiene acceso directo a recursos gubernamentales y a información especializada, a la vez que tiende a cooperar con organizaciones a las cuales puede cooptar. Aparece como un actor menos central que las organizaciones políticas de pobladores, al menos en su capacidad de influencia, 
en tanto su localización queda restringida por la influencia que logró desarrollar el movimiento de pobladores en las comunidades populares, como La Faena y Lo Hermida, y San Luis en los sectores más acomodados, por la acción de los dirigentes de la Jj.vv. de la Comunidad Ecológica ( $\left.n^{\circ} 49\right)$.

Cabe destacar tres cuestiones importantes que deja el análisis de los actores en posiciones de prominencia. En primer lugar, que el movimiento de pobladores es un actor muy relevante en tanto su posición genera una gran influencia política, en la medida en que contribuye a la integración horizontal (Espinoza, 1999a) entre los actores de la sociedad civil, y vertical entre los campos liberal-demócratas y de la política popular. Su prestigio depende de su posición de intermediario, a la vez que esta posición pudo contribuir a la difusión de orientaciones y disposiciones de tipo conflictivo.

Por otro lado, cabe mencionar que actores como las organizaciones dedicadas a la educación popular (OPB), y las vecinales (ov), se hacen más importantes si consideramos la función de centralidad de intermediación y de flujo que cumplen, desplazando en importancia a varias organizaciones de pobladores, como algunos comités de vivienda con una alta centralidad beta. Finalmente, es pertinente insistir en que las tendencias estadísticas parecen ser contradictorias, dado el rol que cumple la Comunidad Ecológica. Su alto grado de intermediación no se traduce en prestigio, debido al carácter estrictamente local de su demanda, pero la alianza con los sectores populares de la comuna significó el acceso mutuo a recursos escasos e importantes para el devenir de la campaña en general.

\section{Sobre la centralidad de los bloques}

Para este apartado utilizados una técnica de detección de equivalencia estructural. En específico usamos el procedimiento CONCOR, a través del cual se pudo detectar cuatro bloques en función de la identificación de pautas de vinculación similares que circunscriben a los actores y definen posiciones sociales. Como detallamos en el apartado metodológico, para este análisis usamos relaciones múltiples (múltiples matrices de datos direccionados y no valorados), lo cual permite una interpretación más sustantiva de los grupos que se conformaron a través de pautas de interacción (figura 3, figura 4, figura 5, figura 6).

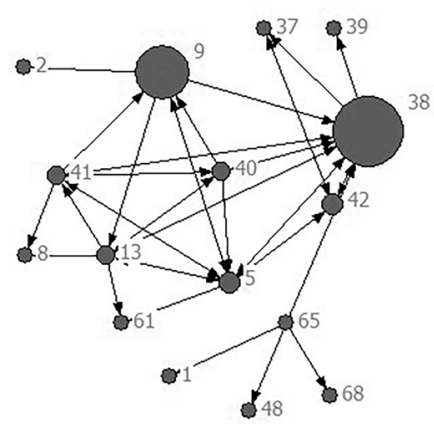

FIGURA 3 | Bloque 1, correspondiente al mundo de acción colectiva ligado a vivienda CV y OPP FUENTE: ELABORACIÓN PROPIA

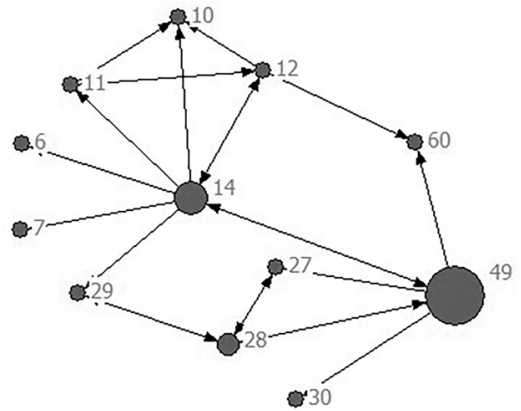

FIgURA 4 | Bloque 2, corresponde a las organizaciones de la elite local, incluida la municipalidad y la JJ.vv. de la Comunidad Ecológica FUENTE: ELABORACIÓN PROPIA 


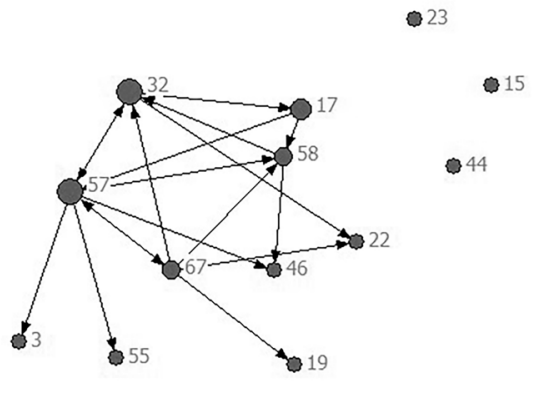

FIGURA 5 | Bloque 3, correspondiente a organizaciones vecinales (ov) y principalmente JJ.Vv.

FUENTE: ELABORACIÓN PROPIA

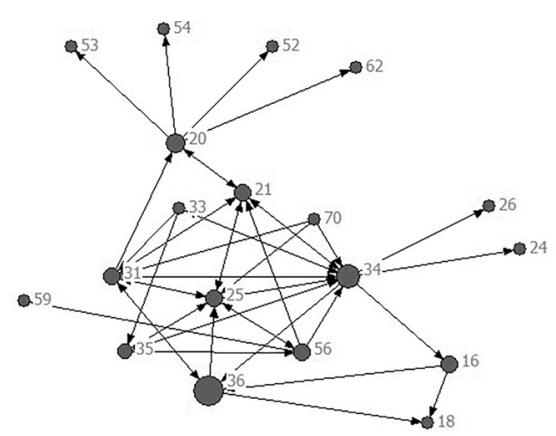

FIGURA 6 | Bloque 4, correspondiente a las organizaciones culturales y políticas de base de sectores populares de Peñalolén (орв, oc) FUENTE: ELABORACIÓN PROPIA

\begin{tabular}{|c|c|c|c|}
\hline \multirow{2}{*}{ BLOQUES } & \multicolumn{2}{|c|}{ CENTRALIDADES } & \multirow{2}{*}{ DENSIDAD } \\
\cline { 2 - 3 } & NOUTDEG & NINDEG & 0,167 \\
\hline 1 & 0,037 & 0,130 & 0,048 \\
\hline 2 & 0,021 & 0,021 & 0,110 \\
\hline 3 & 0,036 & 0,054 & 0,134 \\
\hline 4 & 0,154 & 0,080 & \\
\hline
\end{tabular}

TABLA 2 Centralidades y densidad por bloque identificado FUENTE: ELABORACIÓN PROPIA

\begin{tabular}{|c|c|c|c|c|}
\hline BLOQUES & $\mathbf{I}$ & $\mathbf{2}$ & $\mathbf{3}$ & $\mathbf{4}$ \\
\hline 1 & 0,167 & 0,003 & 0 & 0,003 \\
\hline 2 & 0,003 & 0,048 & 0 & 0 \\
\hline 3 & 0,009 & 0 & 0,110 & 0,008 \\
\hline 4 & 0,014 & 0 & 0,028 & 0,134 \\
\hline
\end{tabular}

TABLA 3 | Densidades dentro y en las relaciones entre bloques FUENTE: ELABORACIÓN PROPIA

\section{La dinámica de movimiento social}

La dinámica del movimiento social (bloques 1 y 4) circunscribe aquellas organizaciones ligadas a las demandas urbanas por vivienda social, y organizaciones comunitarias de tipo cultural y políticas dedicadas a la educación popular, arraigadas en sectores emblemáticos y populares de la comuna (como La Faena, Lo Hermida y San Luis). La tabla 3 nos revela que en el bloque 1 se encuentran las organizaciones del movimiento de pobladores -comités de vivienda (cv) y organizaciones políticas del movimiento (Opp)-, es el bloque más prestigioso y el que posee mayor 
densidad, considerando la del bloque en sí mismo y la densidad de la relación con los demás. Asimismo, fueron estas organizaciones las que dieron vida al Consejo de Movimientos Sociales, el cual nucleó una gran cantidad de comités de vivienda y de organizaciones políticas, como el Movimiento de Pobladores Sin Techo (MPST), el Partido igualdad, el MPL, la FEnAPO. Adicionalmente, en el bloque 4 se desarrolla una dinámica similar: en él se encuentran organizaciones culturales (oc) y las dedicadas a la educación popular (орв). Vale enfatizar que estas fueron particularmente importantes a la hora de integrar el movimiento estrictamente ligado a la vivienda, con la base organizativa de las poblaciones emblemáticas de la comuna. Por su parte, la importancia de las agrupaciones culturales puede radicar en que cumplieron un rol preponderante en las manifestaciones populares, ya sea en cortes de ruta masivos o en marchas/caravanas, y que fueron un eslabón informativo importante para las comunidades. Posiblemente ayudaron a diversificar y ampliar la influencia y legitimidad del repertorio clásico de protesta y tomas de las organizaciones de pobladores. Este bloque en general agrupa los mayores grados de centralidad y densidad dentro y entre los bloques (tablas 2 y 3), lo cual es indicador de su cohesión y prestigio, basados posiblemente en su credibilidad y en acuerdos contingentes sobre aspectos de los problemas de interés común y/o formas de protesta. Esto, a su vez, indica niveles de coherencia e importancia que pueden asumirse como indicadores de emergencia de procesos de identidades colectivas.

Las organizaciones de educación popular (орв) conforman un espectro de actividades que va desde la formación de preuniversitarios populares, trabajo infantojuvenil de prevención, hasta las actividades conmemorativas realizadas año tras ańo. El eslabón representado por el bloque 4 cumple con el objetivo de mantener vivas las historias que actúan como depositarias de significados que dan contenido a la identidad de las comunidades. Generalmente no reciben subvención a sus programas, de modo que muchas no logran trascender debido a la falta de recursos. En este caso, su principal recurso es de tipo reticular, en tanto su capacidad articuladora facilitó procesos de circulación de información e identificación en los sectores más desafectados políticamente.

\section{La dinámica comunitaria}

El bloque 3 es menos denso que los dos anteriores y constituido por organizaciones vecinales (ov) de gobierno de base (JJ.Vv.), incluidos clubes deportivos y organizaciones de adulto mayor. El rol democrático de las JJ.vv. les exige mantener abiertos canales y flujos de comunicación con todo tipo de organizaciones. Aunque la mayoría de las veces las organizaciones vecinales referenciaron a otras circunscritas al mismo territorio y del mismo tipo organizativo, su cercanía y vínculo con organizaciones territoriales dedicadas a la educación popular y las de tipo cultural (la relación entre el bloque 3 y 4 es la más densa), les permitió mantener vínculos lejanos y estratégicos con organizaciones de pobladores, las cuales acudieron a ellas para solicitar diversos tipos de recursos. La identidad política emergente a través de esta estructura de base permite el solapamiento y el soporte territorial y comunitario de las diversas demandas de los pobladores. El conocimiento popular y libretos en 
común (Tilly, 2000), muy presentes en los dirigentes de organizaciones de gobierno de base, como las organizaciones vecinales (JJ.Vv.) y clubes deportivos (CD), facilitaron la conexión de las bases sociales ajenas al movimiento de pobladores con la información especializada producida por este. Es relevante mencionar que estas articulaciones se dan en gran medida debido a que las agrupaciones culturales suelen ser dependientes de las organizaciones vecinales de gobierno de base (JJ.vv.), ya que muchas veces son estas últimas las que ceden espacios y recursos varios para su despliegue cotidiano. En este sentido, la relación entre los bloques 1, 3 y 4 resulta clave. Si bien la densidad del bloque 3 es baja en comparación con las demás, se compensa con su heterogeneidad y alcance territorial. Al mismo tiempo, sostiene una relación lejana pero fluida (gracias a la intermediación del bloque 4) con las organizaciones del movimiento de pobladores.

\section{La coalición}

El bloque 2 está conformado por organizaciones que comparten un mayor acceso a recursos en general. Con la excepción de los comités de vivienda que fueron cooptados por la municipalidad ( $\mathrm{N}^{\circ}$ s. $\left.6,10,11,12\right)$, las demás organizaciones representan un espacio social vinculado a las elites locales en términos políticos y económicos. Al respecto, los vínculos de la red de cooperación de la Municipalidad $\left(\mathrm{n}^{\circ} 14\right)$ con la Comunidad Ecológica $\left(\mathrm{n}^{\circ} 49\right)$ y otras entidades, como las consultoras dedicadas al desarrollo de la propuesta del PRC, algunas ONG, como "Defendamos la ciudad" y el Colegio de Arquitectos de Chile, le permiten el acceso a un gran flujo de información especializada. Este bloque en particular es el menos denso y menos prominente, si consideramos las medidas de centralidad. En breve, parece no haber mucha redundancia en sus flujos comunicativos, lo cual puede ser indicador de baja homogeneidad en sus opiniones y formas de acción, y en general de límites identitarios menos claros, a diferencia de lo que ocurre con los bloques 1 y 4 . Sin embargo, este bloque agrupa actores altamente influyentes, como la JJ.vv. de la Comunidad Ecológica. La alianza interclase con organizaciones de pobladores y de sectores populares en general que representa este actor $\left(n^{\circ} 14\right)$, muestra una división de la elite local, al mismo tiempo que una oportunidad para las organizaciones populares.

A modo de síntesis, podemos destacar dos aspectos. En primer lugar, que los bloques más prominentes y cohesionados son el 1 y el 4 . En los hechos, ambos compartieron una identidad en un lado de la contienda, y su conexión redunda en la integración de entramados mayores, toda vez que las organizaciones de gobierno de base están densamente conectadas al bloque 4. Adicionalmente, es en la relación de estos bloques donde tomó forma un modo de coordinación de movimiento social altamente influyente para el devenir de la contienda.

\section{Conclusiones}

En un escenario político local altamente fragmentado, las lecturas actuales de los movimientos urbano-populares no le han dado suficiente importancia al análisis de los mecanismos y relaciones que modifican la configuración de la sociabilidad popular para facilitan el poder de las clases subordinadas. Una perspectiva de redes 
puede, entonces, aportar en la tarea de analizar la constitución de identidades políticas en los conflictos urbanos del Chile neoliberal.

Con el análisis de la centralidad de los actores y de posiciones estructurales, pudimos comprender de mejor manera cómo se construyó una estructura de poder altamente influenciada por el movimiento de pobladores, la cual permitió el freno a una iniciativa de una elite local dirigida por la Municipalidad de Peñalolén. A nivel de actores, pudimos corroborar que las organizaciones de pobladores, al menos en este episodio, actuaron como intermediarios horizontales de integración de entramados lejanos. A nivel de bloques, sus relaciones nos permiten visualizar de mejor manera la alianza del llamado Consejo de Movimientos Sociales con organizaciones comunitarias de diversos territorios y sectores socioeconómicos, a través de vínculos clave entre organizaciones prominentes y diversas, lo cual permitió que sectores populares y mayormente desaventajados tuvieran mayores probabilidades de influencia sobre la política pública de regulación de recursos urbanos.

\section{Agradecimientos}

Al Centro de Estudio del Conflicto y la Cohesión Social (coes), Conicyt/ Fondap/15130009, y a los proyectos Fondecyt 1171426 y 1160984, por el financiamiento otorgado durante el desarrollo de la investigación que sustenta estos análisis.

\section{Referencias bibliográficas}

Angelcos, N. S. (2016). Movimiento de pobladores. Lucha social y política en el Chile contemporáneo. Educação em Perspectiva, 7(2). https://doi.org/10.22294/eduper/ ppge/ufv.v7i2.788

Angelcos, N. \& Pérez, M. (2017). De la "desaparición” a la reemergencia: Continuidades y rupturas del movimiento de pobladores en Chile. Latin American Research Review, 52(1), 94-109. https://doi.org/10.25222/larr.39

Banco Mundial (2013). Informe sobre el desarrollo mundial 2014. Panorama general: Riesgo y oportunidad. La administración del riesgo como instrumento de desarrollo. Washington, DC: Banco Mundial. Licencia: Creative Commons. Reconocimiento CC BY 3.o. https:// ibce.org.bo/images/publicaciones/Informe-sobre-desarrollo-mundial.pdf

Barozet, E. (2016). Entre la urna, las redes y la calle: Las relaciones entre movimientos sociales y partidos políticos en el Chile democrático. En M. A. Garretón Merino (ed.), Ciencias sociales y humanas. Ciencias politicas. La gran ruptura: institucionalidad politica y actores sociales en el Chile del siglo XXI (pp. 21-58). Santiago de Chile: LOM Ediciones.

Bolland, J. (1988). Sorting out centrality: An analysis of the performance of four centrality models in real and simulated networks. Social Networks, 10(3), 233-253. https://doi. org/10.1016/0378-8733(88)90014-7

Bonacich, P. (1987). Power and centrality: A family of measures. American Journal of Sociology, 92(5), 1170-1182. https://doi.org/10.1086/228631 
Borgatti, S. (2002). NetDraw Software for Network Visualization. Lexington, KY: Analytic Technologies.

Borgatti, S., Everett, M., \& Freeman, L. (2002). Ucinet for windows: Software for social network analysis. Harvard, MA: Analytic Technologies.

Borgatti, S., Everett, M., \& Johnson, J. (2018). Analyzing social networks. London, uk: Sage.

Burt, R. (1977). Power in a social topology. Social Science Research, 6(1), 1-83. https://doi. org/10.1016/0049-089X(77)90001-1

Carrington, P., Scott, J., \& Wasserman, S. (2005). Models and methods in social network analysis. Cambridge, uk: Cambridge University Press.

Castells, M. (2005). Globalización, desarrollo y democracia: Chile en el contexto mundial (1 $\left.1^{\mathrm{a}} \mathrm{ed}\right)$. Santiago: Fondo de Cultura Económica Chile.

Castells, M. (2008). La cuestión urbana. (17a ed.). México, DF: Siglo XXI.

Cortés, A. (2014). El movimiento de pobladores chilenos y la población La Victoria: Ejemplaridad, movimientos sociales y el derecho a la ciudad. EURE, 40(119), 239260. http://doi.org/10.4067/S0250-71612014000100011

Crossley, N. (2011). Towards relational sociology. Abingdon, UK: Routledge.

Davis, M. (2014). Planeta de ciudades miseria. Madrid: Akal.

De la Maza, G. (2010). Construcción democrática, participación ciudadana y políticas públicas en Chile (Tesis doctoral). Universiteit Leiden. https://bit.ly/2M9fnpx

Diani, M. (2003). Leaders or brokers? positions and influence in social movement networks. En M. Diani \& D. McAdam (eds.), Social movements and networks: Relational approaches to collective action (pp. 105-122). Nueva York: Oxford University Press. http://doi.org /10.1093/0199251789.003.0005

Diani, M. (2015). The cement of civil society: Studying networks in localities. Nueva York: Cambridge University Press.

Diani, M. \& Bison, I. (2004). Organizations, coalitions, and movements. Theory and Society, 33(3-4), 281-309. https://doi.org/10.1023/B:RYSO.0000038610.00045.07

Domínguez, S. \& Hollstein, B. (eds.) (2014). Mixed methods social networks research: Design and applications (Vol. 36). Nueva York: Cambridge University Press.

Doreian, P., Batagelj, V., \& Ferligoj, A. (2005). Generalized blockmodeling (Vol. 25). Nueva York: Cambridge University Press.

Espinoza, V. (1988). Para una historia de los pobres de la ciudad. Santiago: Ediciones SUR.

Espinoza, V. (1995). Redes sociales y superación de la pobreza. Revista de Trabajo Social (66), $31-44$.

Espinoza, V. (1999a). Continuidad histórica de la acción colectiva de los pobladores chilenos. redes sociales e interacción estratégica. En T. Salman \& E. Kingman (eds.), Antigua modernidad y memoria del presente. Culturas urbanas e identidad (pp. 189-217). Quito: Flacso-Ecuador.

Espinoza, V. (1999b). Social networks among the urban poor: inequality and integration in a Latin American city. En B. Wellman (ed.), Networks in the global village: Life in contemporary communities (pp. 147-184). Boulder, co: Westview Press.

Espinoza, V. (2013). Local associations in Chile: Social innovation in a mature neoliberal society. En F. Moulaert, D. MacCallum, A. Mehmood, \& A. Hamdouch (eds.), The International Handbook on Social Innovation. Collective action, social learning and transdisciplinary research (pp. 397-411). https://doi.org/10.4337/9781849809993.00048 
Everett, M. \& Borgatti, S. (1999). The centrality of groups and classes. The Journal of Mathematical Sociology, 23(3), 181-201. https://doi.org/10.1080/0022250X.1999.9990219

Friedkin, N. (2006). A structural theory of social influence (Vol. 13) Nueva York: Cambridge University Press.

Garretón, M. (2001). Cambios sociales, actores y acción colectiva en América Latina. Serie Políticas Sociales 56 [LC/L.I608-P]. Santiago: Comisión Económica para América Latina y el Caribe (CEPAL). https://repositorio.cepal.org/handle/11362/6012

Gasic, I. (2018). Inversiones e intermediaciones financieras en el mercado del suelo urbano. principales hallazgos a partir del estudio de transacciones de terrenos en Santiago de Chile, 2010-2015. EURE, 44(133), 29-50. http://doi.org/4067/s025071612018000300029

Gould, R. \& Fernandez, R. (1989). Structures of mediation: A formal approach to brokerage in transaction networks. Sociological Methodology, 19, 89-126. http://doi. org/10.2307/270949

Harvey, D. (2007). Urbanismo y desigualdad social. Madrid: Siglo XxI.

Herrera, J. (2018). El nuevo movimiento de pobladores en Chile: El movimiento social desplazado. POLIS, Revista Latinoamericana, 17(49), 177-199. http://doi.org/10.40 67/S0718-65682018000100177.

Imilan, W. (2016). Políticas y luchas por la vivienda en Chile: el camino neoliberal. Working paper series CC. contested_cities 16004. http://repositorio.uchile.cl/bitstream/2250/141198/1/ Politicas-y-luchas-por-la-vivienda-en-chile-el-camino-neoliberal.pdf

Knoke, D. (1994). Political networks: The structural perspective (Vol. 4) Nueva York: Cambridge University Press.

Knoke, D. \& Burt, R. (1983). Prominence. En R. S. Burt \& M. J. Minor (eds.), Applied network analysis: a methodological introduction (pp. 195-222). Beverly Hills, CA: Sage.

Lin, N. (2002). Social capital: A theory of social structure and action. Nueva York: Cambridge University Press.

Lomnitz, L. (2008). Lo formal y lo informal en las sociedades contemporáneas. Santiago de Chile: Centro de Investigaciones Diego Barros Arana.

Merklen, D. (2010). Pobres ciudadanos: Las clases populares en la era democrática (Argentina, 1983-2003) (2 ed.). Novecento: Vol. 19. Buenos Aires: Gorla.

Oxhorn, P. (2001). Desigualdad social, sociedad civil y los límites de la ciudadanía en América Latina. Economía Sociedad y Territorio. https://doi.org/10.22136/est002001368

Özler, Ş. (2012). The Concertación and homelessness in Chile: Market-based housing policies and limited popular participation. Latin American Perspectives, 39(4): Michelle Bachelet: The last Concertación government, 53-70. https://doi.org/10.1177 /0094582X10397917

Pérez, M. (2017). "A new poblador is being born": Housing struggles in a gentrified area of Santiago. Latin American Perspectives, 44(3), 28-45. https://doi.org/10.1177/ $0094582 X 16668318$

Poduje, I. (2008). Participación ciudadana en proyectos de infraestructura y planes reguladores. Temas de la Agenda Pública. Santiago: P. Universidad Católica de Chile. https:// repositorio.uc.cl/bitstream/handle/11534/1521/514662.pdf?sequence $=1$ 
Pulgar, C. (2012). El doble movimiento telúrico y social: Chile después del terremoto del 27 de febrero de 2010. Movimientos sociales urbanos, ciudad neoliberal, reconstrucción, justicia espacial y derecho a la ciudad. París: Ecole des hautes études en sciences sociales de Paris.

Rosanvallon, P. (2012). La sociedad de iguales. Buenos Aires: Manantial.

Scott, J. (2007). Power, domination and stratification: Towards a conceptual synthesis. Sociologia, Problemas e Práticas, (55), 25-39. https://repositorio.iscte-iul.pt/bitstream /10071/1112/1/2.pdf

Scott, J. (2011). Conceptualising the social world: Principles of sociological analysis. Nueva York: Cambridge University Press.

Skewes, J. (2001). La exacerbación de la desigualdad en la periferia urbana en Santiago, Chile. El diseño espacial de los asentamientos irregulares y su desmantelamiento a través de las políticas de vivienda Fermentum. Revista Venezolana de Sociología y Antropología, 11(31), 256-272. http://www.redalyc.org/articulo.oa?id=70511242006

Svampa, M. (2008). Cambio de época. Buenos Aires: Siglo xxi.

Tilly, C. (2000). La desigualdad persistente. Buenos Aires: Manantial.

Tilly, C. (2010). Democracia. Madrid: Akal.

Tilly, C., Tarrow, S., \& McAdam, D. (2005). Dinámica de la contienda politica. Barcelona: Hacer.

Wasserman, S. \& Faust, K. (2013). Análisis de redes sociales. métodos y aplicaciones. Madrid: Centro de Investigaciones Sociológicas.

White, D. R. (1994). Enfoque de redes para el estudio de comunidades urbanas. Estudios Demográficos y Urbanos, 9(2), 303-326. http:// doi.org/10.24201/edu.v9i2.909

White, H. C. (2008). Identity and control: How social formations emerge ( $2^{\mathrm{a}}$ ed.). Princeton, NJ: Princeton University Press. 